\title{
Changes in the Cell Membrane of Erythrocytes in Tumor-Bearing Rats
}

\author{
Tatsuzo Fujiii, Kinya Koizumi ${ }^{2}$, Keiko Tamiya-Koizumi ${ }^{3}$ and \\ Kiyohide Kojima ${ }^{3}$ \\ ${ }^{1}$ Department of Biochemistry, Kyoto Pharmaceutical University, Kyoto 607, \\ ${ }^{2}$ Central Research Laboratories, Chugai Pharmaceutical Co. Ltd., Tokyo 171, \\ and ${ }^{3}$ Research Institute for Disease Mechanism and Control, Nagoya University \\ School of Medicine, Nagoya 466, Japan
}

\begin{abstract}
Cell electrophoresis of erythrocytes taken from AH 7974 hepatoma-bearing rats showed a marked decrease in the surface negative charge, as represented by a decrease in cell mobility. This change in the erythrocyte membrane began during the stationary phase of growth of transplanted tumor cells and became more pronounced in the late phase, during which time a marked decrease in the amount of sialidase-sensitive sialic acid present in the plasma membrane also took place. The erythrocytes of AH 7974F-bearing rats (which died from tumor growth much earlier than the AH 7974-bearing rats) had a similar, but less marked, decrease in their electrophoretic mobility and in the amount of sialidase-sensitive sialic acid present. These erythrocytes all had somewhat abnormal cell morphology, their shapes being slightly spherocytic.
\end{abstract}

In our studies of the cell membrane of rat ascites hepatoma cells, we found spherocytic erythrocytes (Fig. 1) in the peripheral blood and ascitic fluid of hepatomabearing rats. Because a change in the shape of erythrocytes often reflects an abnormality in the cell membrane (3), we investigated possible alterations in the composition and properties of the erythrocyte membrane.

The tumor-bearing rats used in the experiments reported here were prepared by intraperitoneal transplantation of Yoshida ascites hepatoma AH 7974 (an islandforming line) or AH 7974F (a free-cell type subline of AH 7974) in inbred Moriyama strain rats, as reported elsewhere (4). Tumor cell growth in rat ascitic fluid as a function of time is depicted in Fig. 2.

Blood was collected in a heparinized syringe by heart puncture. Erythrocytes were separated by centrifugation, then washed and suspended in $67 \mathrm{mM}$ phosphate buffer supplemented with $5.4 \%$ glucose (pH 7.3, ionic strength 0.167 ). Next, the cells were electrophoresed with a cytopherometer (Zeiss) at $25 \pm 0.5^{\circ} \mathrm{C}(5,6)$.

The sialic acid content of the cells was determined by Aminoff's thiobarbituric acid method (1). Sialidase-sensitive sialic acid in the plasma membrane (6) was determined by incubating the intact erythrocytes at $37^{\circ} \mathrm{C}$ for 30 min with 20 units per $\mathrm{ml}$ of neuraminidase (sialidase) preparation obtained from Vibrio cholerae (General Biochemicals, Inc.) (5). The membrane lipids were assayed as described previously (7). 

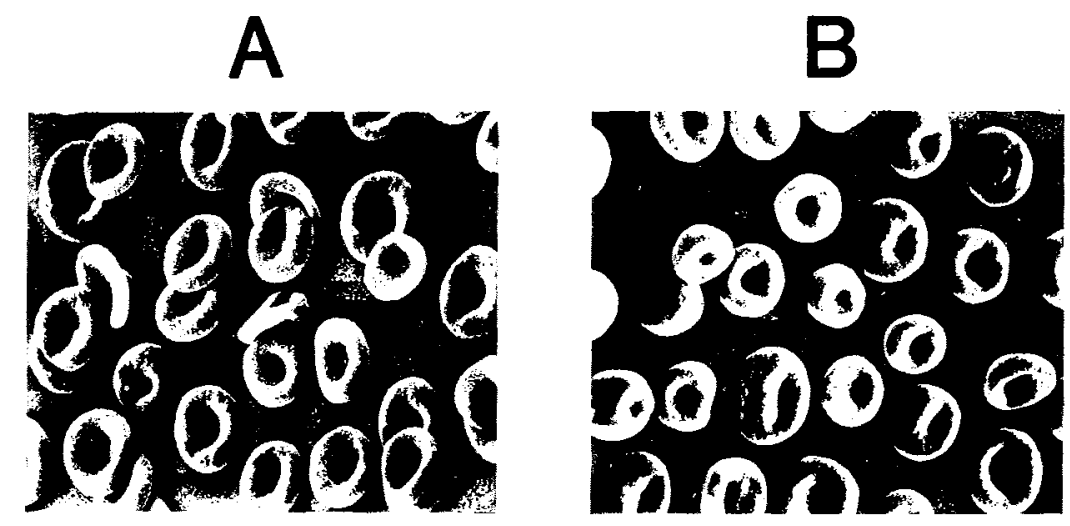

Fig. 1. Scanning electron micrographs of erythrocytes taken from normal (A) and AH 7974bearing rats $(\mathrm{B})$. Magnification, $\times 1000$.

The electrophoretic mobility of the erythrocyte cell population taken from $\mathrm{AH}$ 7974-bearing rats at the exponential, stationary and late phases of tumor growth (4, 8 and 12 days after transplantation) is shown in Fig. 3-B, -C and -D, and is compared with that of a normal erythrocyte population (Fig. 3-A) from control rats. Four days after transplantation, the erythrocytes showed a slight increase in mobility. This may have been due to increased number of reticulocytes, which have higher mobility than mature erythrocytes, at the stage when acute anemia occurs. Eight days after transplantation, the erythrocytes showed a marked decrease in mobility, which represented the reduced negative charge of the cell membrane surface. This change

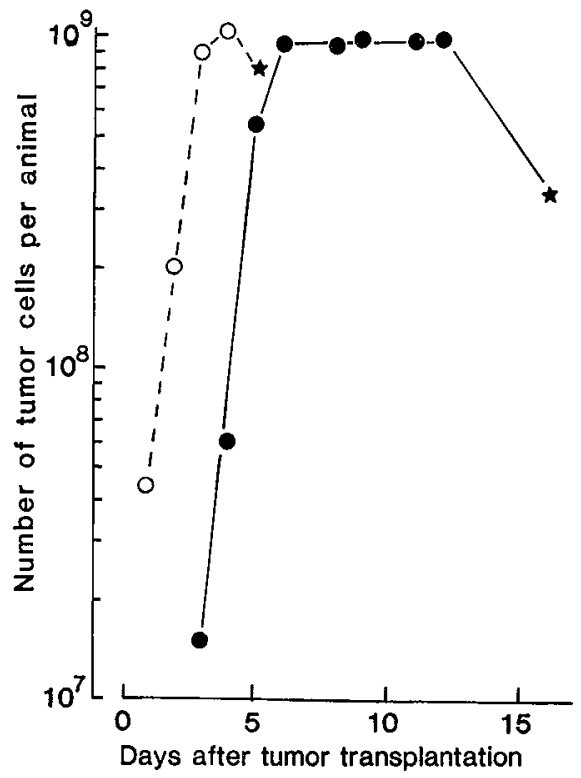

Fig. 2. Typical time course of tumor cell growth in tumor-transplanted rats. Ascites hepatoma AH $7974(\bullet)$ and AH 7974F (O). The star indicates the death of the host animal. 


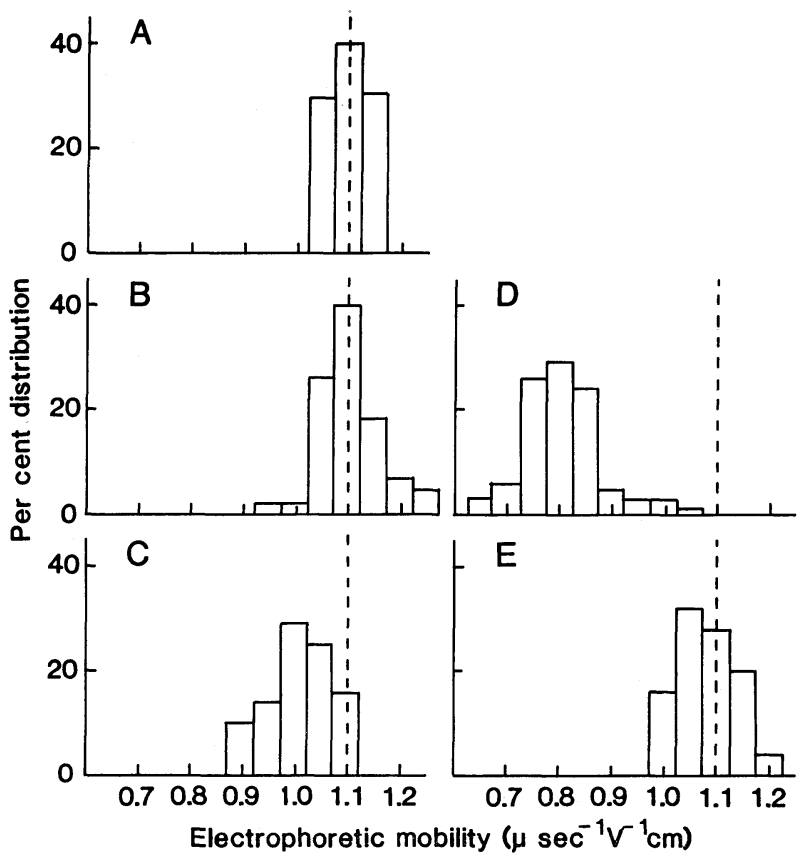

Fig. 3. Typical distribution pattern of electrophoretic mobility in an erythrocyte population of tumor-bearing and normal rats. A: erythrocytes from a normal rat, B-D: from an AH 7974-bearing rat and $\mathrm{E}$ : from an $\mathrm{AH} 7974 \mathrm{~F}$-bearing rat. In B and $\mathrm{C}$, erythrocytes were taken from the rats when tumor growth was in the exponential and stationary phases, 4 and 8 days after transplantation. In $\mathrm{D}$ and $\mathrm{E}$, erythrocytes were taken from rats when tumor growth was in the late phase, 12 days after transplantation for AH 7974 and 4 days after for $\mathrm{AH} 7974 \mathrm{~F}$ animals. The dotted line shows the median mobility distribution in the normal erythrocyte population.

was more prominent 12 days after transplantation. A similar but less obvious tendency was found in the erythrocytes of AH 7974F-bearing rats in the late phase of growth (4 days after transplantation) (Fig. 3-E).

As the sialidase-sensitive sialic acid in the erythrocyte membrane is the main acidic sugar residue that determines the electrophoretic mobility of the cell (6), we assayed the sialic acid content of the erythrocytes in tumor-bearing rats during the late phase

TABLE 1. SiALIC ACID CONTENT OF ERYTHROCYTES FROM NORMAL AND TUMOR-BEARING RATS

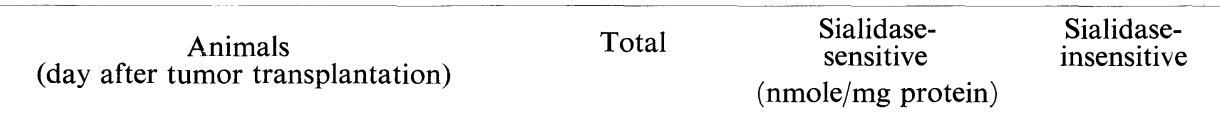

$\begin{array}{lccc}\text { Normal rats } & 80.94 & 43.08 & 37.86 \\ \text { AH 7974-bearing rats (12 days) } & 58.65 & 16.28 & 42.37 \\ \text { AH 7974F-bearing rats (4 days) } & 76.04 & 33.13 & 42.91\end{array}$

Each value is the mean of two determinations. 
of the tumor growth. The amount of sialidase-sensitive sialic acid residues released by sialidase treatment was markedly lower in cells of tumor-bearing rats than those of normal rats, whereas the sialidase-insensitive portion remained virtually unaltered (Table 1). Erythrocytes from the AH 7974-bearing rats showed a greater decrease in the amount of sialic acid present than did those of the AH 7974F-bearing rats. An alteration in the surface sialic acid content of the membrane apparently is the explanation of the change in the electrophoretic mobility of the erythrocytes.

Lipid analysis showed no marked difference in the total phospholipid and cholesterol contents or in the phospholipid composition of erythrocytes taken from the tumor-bearing and control animals (data not shown).

The abnormalities found in relation to the cell membrane (reduced electrophoretic mobility and reduced sialic acid content) may be related, but what the relation of these phenomena to the altered shape of the cell membrane is, has yet to be established.

We speculate that these alterations are caused by particular enzyme(s) or another factor(s) released from a necrotic portion of the tumor. Chandler and Fletcher (2) attributed the abnormal morphology of the surface membrane of erythrocytes (including pits and craters) taken from chicken with a transplanted lymphoid tumor to the action of such a released factor(s). The fact that erythrocytes of AH 7974Fbearing rats in the late phase of tumor growth showed less of a decrease in their electrophoretic mobility and sialic acid content than did those of AH 7974-bearing rats may be due to insufficient time for the release of such a factor in animals that showed maximal tumor growth by 3 to 4 days after transplantation (Fig. 2). In contrast, AH 7974-bearing rats showed maximal tumor growth from 6 to 12 days after transplantation (Fig. 2); therefore, there was sufficient time for the release of such a factor. These discussions are, however, purely speculative. The cause of the abnormalities present in the erythrocytes of tumor-transplanted rats requires further investigation.

\section{REFERENCES}

1. Aminoff, D. Methods for the quantitative estimation of $N$-acetylneuraminic acid and their application to hydrolysates of sialomucoids. Biochem. J. 81, 383-392, 1961

2. Chandler, F.W. and O.J. Fletcher. Erythrocyte membrane defects associated with a transplantable lymphoid tumor. J. Natl. Cancer Inst. 51, 1351-1353, 1961

3. FUנII, T. The plasma membrane and cell shape (in Japanese). Maku(Membrane) 1, 82-92, 1976

4. Koizumi, K., K. Tamiya-Koizumi, T. FujII and K. Kojima. Phospholipids of plasma membrane isolated from rat ascites hepatomas and from normal rat liver. Cell Struct. Funct. 2, $145-153,1977$

5. Sato, C. and K. Kojima. Changes in electrophoretic mobility of cultured cells after X-irradiation and their modification by SH-blocking agents and hemagglutinin. Radiat. Res. 60, 506515,1974

6. Sato, C., K. Kojima, K. Nishizawa and K. Sato. Dislocation of sialic acid on erythrocyte membrane by anesthetics and its blocking by SH-reagents, colchicine or cytochalasin B. Cell Struct. Funct. 3, 145-151, 1978

7. Sato, T. and T. FusII. Phospholipid distribution in erythrocytes and plasma of man, cow, pig and rabbit. Chem. Pharm. Bull. 19, 377-384, 1971

(Received for publication, September 17, 1984) 